\title{
Trends in dietary carbohydrate quality during puberty from 1988 to 2007: a cause for concern?
}

\author{
Guo Cheng*, Lars Libuda, Nadina Karaolis-Danckert, Ute Alexy, Katja Bolzenius, Thomas Remer \\ and Anette E. Buyken \\ Research Institute of Child Nutrition, Rheinische Friedrich-Wilhelms-Universität Bonn, Dortmund, Germany
}

(Received 19 November 2009 - Revised 25 March 2010 - Accepted 4 May 2010 - First published online 1 July 2010)

The extent to which the quality of dietary carbohydrates (CHO) changes throughout puberty is not known. We analysed trends in the quantity and quality of $\mathrm{CHO}$ intake among German adolescents by separately examining trends during puberty (pubertal trends) and trends in $\mathrm{CHO}$ intake from 1988 to 2007 (secular trends). Linear mixed-effects regression analyses were performed in 216 participants of the Dortmund Nutritional and Anthropometric Longitudinally Designed Study who had provided weighed $3 \mathrm{~d}$ dietary records at the onset of the pubertal growth spurt (defined by age at take-off) and over the subsequent 4 years. Over the course of puberty, CHO quality changed little: added sugar intake from beverages increased in girls $(0 \cdot 25$ (SE 0.12$) \%$ energy (\% E)/year, $P=0.04)$ and added sugar intake from sweets decreased in both sexes (boys: -0.22 (SE 0.11) \% E/year, $P=0.049$; girls: -0.20 (SE 0.10) \% E/year, $P=0 \cdot 04$ ). For both sexes, significant upward secular trends were observed for CHO (\% E), glycaemic load (g/MJ) and added sugar intakes from sources other than sweets and soft drinks (\% E), while absolute fibre intake $(\mathrm{g} / \mathrm{d})$ decreased $(P \leq 0.04)$. Concomitant increases in total added sugar intake $(\% \mathrm{E})$ and decreases in fibre and whole-grain densities $(\mathrm{g} / \mathrm{MJ})$ $(P=0.001-0.02)$ were confined to boys only. The quality of dietary CHO consumed by healthy German adolescents shows notable secular declines, but does not change markedly during puberty. Public health initiatives should be tailored to improve the overall quality of $\mathrm{CHO}$ nutrition.

Carbohydrate intake: Dietary glycaemic index: Dietary glycaemic load: Fibre intake: Whole-grain intake: Added sugar intake: Adolescents: Pubertal trends: Secular trends

Recent studies indicate notable changes in dietary quality from childhood to adolescence. Consumption of fruits and vegetables has been found to decrease ${ }^{(1)}$, whereas the consumption of soft drinks increased ${ }^{(1)}$. Furthermore, as children age, they increase their fast food consumption ${ }^{(2)}$, which in turn has been found to be associated with lower intakes of fruits, vegetables and grains, and with higher sugar intakes ${ }^{(3)}$. It is thus intriguing to consider that carbohydrate $(\mathrm{CHO})$ quality in particular declines as puberty commences, but this has not been investigated to date.

In addition, concern has been expressed that recent increases in relative $\mathrm{CHO}$ intake among European and North American children and adolescents ${ }^{(4,5)}$ may have been accompanied by a secular decline in $\mathrm{CHO}$ quality. In fact, prospective studies indicate that increases in $\mathrm{CHO}$ intake were mostly due to a higher consumption of pastries/cakes and sweets without an accompanying increase in fibre intakes ${ }^{(6,7)}$.

Considering that puberty may represent the so-called 'critical period' for the development of overweight ${ }^{(8)}$, it is important to disentangle pubertal and secular trends in the quality of CHO intake in order to develop preventive strategies specifically targeted at adolescents. Preferably, such investigations should take pubertal stage into account, since individual changes in food intake may to some extent reflect adaptations to changes in physiological requirements during puberty.

Using data obtained from the Dortmund Nutritional and Anthropometric Longitudinally Designed (DONALD) Study, the objective of the present analysis was to analyse individual changes in CHO quality - characterised by glycaemic index (GI), glycaemic load (GL), and fibre, whole-grain and added sugar intakes - during puberty (between puberty onset and the subsequent 4 years) (pubertal trend), and to separate this trend from changes in $\mathrm{CHO}$ quality which may have occurred between 1988 and 2007 (secular trend) in German adolescents.

\section{Materials and methods}

Study sample

The DONALD Study is an ongoing, open cohort study that was started in 1985 in Dortmund, Germany. Details have been described elsewhere ${ }^{(9)}$. The study was approved by the ethics committee of the University of Bonn, and all examinations were performed with parental consent.

Abbreviations: ATO, age at take-off; CHO, carbohydrates; DONALD Study, Dortmund Nutritional and Anthropometric Longitudinally Designed Study; GI, glycaemic index; GL, glycaemic load.

* Corresponding author: G. Cheng, fax +49 2317115 81, email cheng@fke-do.de 
For the purpose of the present analysis, changes in dietary $\mathrm{CHO}$ quality during physiologically defined puberty were of interest. Chronological age may be confounded by children of the same age differing considerably in their pubertal stage. Defining puberty based on visual observation according to Tanner stage has been criticised as being subjective with considerable inter-observer variability ${ }^{(10)}$, and is commonly perceived as intrusive by healthy individuals ${ }^{(11)}$. Conversely, puberty can be more objectively determined from serial height measurements, an approach chosen in several longitudinal studies $^{(12-14)}$. We thus defined puberty onset as the age at take-off (ATO), i.e. the beginning of the pubertal growth spurt.

In total, ATO could be reliably estimated for 376 subjects. Of these, 216 adolescents who had complete anthropometric and nutritional data around ATO and information on potential confounders were included in the present analysis. Plausible checks according to the age- and sex-specific cut-off points ${ }^{(15)}$ revealed implausible energy intake in 88 of the 1053 dietary records. Since the energy intakes of these eighty-eight dietary records ranged between $3347.2 \mathrm{~kJ}(800 \mathrm{kcal})$ and $9414 \mathrm{~kJ}$ $(2250 \mathrm{kcal})$, we retained all records so as to base our intraindividual trend analyses on as many repeatedly collected diaries as possible. Due to the open cohort design of the DONALD Study, participants reached ATO between 1988 and 2003, and all of them attained their peak height velocity within 4 years of puberty onset, i.e. all our subjects reached an advanced stage of puberty within the present study period.

\section{Anthropometry and assessment of puberty onset}

Anthropometric measurements are performed at each visit by nurses who have been trained according to standard procedures $^{(16)}$, with the children dressed in underwear only and barefoot. From the age of 2 years onwards, standing height is measured to the nearest $0.1 \mathrm{~cm}$ using a digital stadiometer (Harpenden, Crymych, UK).

ATO was estimated using the parametric Preece \& Baines model $1^{(17)}$, based on height measurements from the age of 6 years onwards in boys and on height measurements from the age of 5 years onwards in girls ${ }^{(18)}$. The mean ATO in our sample of 10.3 (SD 0.9) years for boys and 8.7 (SD 0.9) years for girls was in line with mean ATO values reported from other contemporary studies conducted among American and European children ${ }^{(12,19-21)}$.

\section{Nutrition assessment}

Food consumption in the DONALD Study is assessed using $3 \mathrm{~d}$ weighed dietary records ${ }^{(9)}$. For the present analysis, each CHO-containing food recorded in the dietary record was assigned a GI. Foods were assigned either (1) a published $\mathrm{GI}^{(22)}$; (2) the GI of a close match or (3) the GI calculated from the GI values of the foods' ingredients using recipes available in the in-house database Lebensmitteltabelle $(\text { LEBTAB })^{(23)}$. Foods containing mainly fat or protein with a $\mathrm{CHO}$ content below $5 \mathrm{~g} / 100 \mathrm{~g}$ were assigned a GI of 0 (for example, cold meats) ${ }^{(24)}$.

Dietary fibre content was calculated using the LEBTAB database. Whole-grain intake was estimated by assigning whole-grain content in grams to each $\mathrm{CHO}$-containing food recorded in the dietary records using the respective recipes and ingredient information available at the time of recording. In the present analysis, we revised the definition of whole grain used in a previous analysis ${ }^{(25)}$ to only include cereal grains, which contain the same relative proportions of bran, germ and endosperm as they exist in the intact caryopsis ${ }^{(26)}$.

Added sugar intake was defined as described previously ${ }^{(27)}$. Since an increased soft drink consumption during adolescence ${ }^{(2,28)}$ is thought to place children at risk of excess weight gain ${ }^{(29)}$, three subgroups of added sugar intakes were also examined: added sugar from beverages (i.e. from regular soft drinks and fruit juices), added sugar from sweets (i.e. from candy, chocolate, jam and ice cream) and added sugar from other sources (i.e. the remainder of food sources, e.g. breakfast cereals, pastries, milk and milk products).

The GL in the present study correlated with CHO intake ( $r 0.97$ and 0.96 in boys and girls, respectively), GI ( $r 0.38$ and 0.32 in boys and girls, respectively) and added sugar intake ( $r \quad 0.71$ and 0.69 in boys and girls, respectively). We did not assess the validity of the recorded $\mathrm{CHO}$ intakes, since validation of weighed dietary records - commonly considered to be the 'gold standard' method among dietary assessment methods ${ }^{(30)}$ - requires the use of a widely accepted recovery biomarker, which presently does not exist for $\mathrm{CHO}$ intake. However, a recent analysis among DONALD participants has shown an acceptable validity of protein intake data compared with $24 \mathrm{~h}$ urinary nitrogen excretion in a large sample of both children and adolescents ${ }^{(31)}$.

\section{Statistical analysis}

To obtain the mean daily GI and GL values of each subject, we firstly multiplied the CHO content (in g) of each food consumed by the food's assigned GI. The sum of these GL values of each food corresponds to the total GL. The total GI was obtained by dividing the total GL by the total $\mathrm{CHO}$ intake. The mean daily added sugar, fibre or whole-grain intake was the sum of the added sugar, fibre or whole-grain content of each food consumed on each day.

$\mathrm{SAS}^{\circledR}$ procedures (version 9.1.3; SAS, Inc., Cary, NC, USA) were used for data analysis. Analyses indicated interactions between sex and changes in $\mathrm{CHO}$ quality during puberty $(P$ value for interaction $<0 \cdot 1$ ). Thus, data obtained from girls and boys were analysed separately. A $P$ value $<0.05$ was considered to indicate statistical significance.

As suggested by Jacobs et al. ${ }^{(32)}$, repeated-measures regression models (PROC MIXED), including both fixed and random effects, were used to construct longitudinal models of trends in $\mathrm{CHO}$ quality partitioned into pubertal trends and secular trends. The pubertal trend in our models was estimated from the pubertal age coefficient, and the time coefficient represented the secular trend. Separate models for each $\mathrm{CHO}$ variable (the dependent variable) regressed the respective dietary measurement at each examination on pubertal age at examination (ATO was considered the baseline pubertal age, i.e. pubertal age $=0$ ) and the year of examination (first calendar year of the DONALD Study was considered the baseline time, i.e. time $=0$ ).

A repeated statement was used to account for the lack of independence that exists between repeated observations on the same person, and a family variable was used as a random effect. In the unadjusted model, pubertal age and 
time were the principal fixed effects. In the adjusted model, we included BMI standard deviation scores at ATO (to control for potential confounding due to a relation between $\mathrm{CHO}$ quality and BMI standard deviation scores at $\mathrm{ATO}^{(25)}$ ), maternal overweight and maternal education (to account for potential confounding by an association between socio-economic status and dietary choices $\left.{ }^{(33)}\right)$.

\section{Results}

Study sample characteristics at ATO by calendar year strata are presented in Table 1. Between 1988 and 1997, ATO occurred at the age of 10.2 years in boys and 8.6 years in girls, and between 1998 and 2003 at the age of $10 \cdot 3$ years in boys and 8.8 years in girls.

Table 2 summarises mean nutritional intakes of boys stratified by pubertal age and time. Since energy intakes increased notably throughout puberty, the absolute intakes of all measures describing $\mathrm{CHO}$ intake also increased during puberty apart from the intakes of whole grain and added sugar from sweets (first set of columns). With respect to relative measures of $\mathrm{CHO}$ intake, GL and the percentage of energy from other sources increased throughout puberty, while energy from added sugar from sweets decreased. We also observed a significant secular increase in energy intake, and absolute intakes of all $\mathrm{CHO}$ quality measures increased, except for the intakes of whole grain, fibre and added sugar from sweets (second set of columns). With respect to relative intakes, we found secular increases in GL and energy from total added sugar and added sugar from other sources and $\mathrm{CHO}$, and secular decreases in whole-grain and fibre intake densities.

Similarly, energy intake in girls (Table 3) also increased throughout puberty, resulting in increases of all measures describing absolute CHO intake except for the intakes of whole grain and added sugar from beverages and from sweets (first set of columns). Relative $\mathrm{CHO}$ intakes did not change during puberty. Secular increases were observed for GL and absolute intakes of added sugar from sweets and from other sources (second set of columns). Concurrently, absolute fibre intakes decreased, and absolute whole-grain intakes tended to decrease. With respect to relative measures of CHO intake, GI, GL and intakes of added sugar from other sources and $\mathrm{CHO}$ showed secular increases, while intakes of fibre and added sugar from sweets decreased.

Repeated-measures analysis confirmed upward pubertal trends in the absolute intakes of all $\mathrm{CHO}$ intake variables, except for added sugar from beverages in boys and whole grain and added sugar from sweets in both sexes (Table 4, first set of columns). With respect to secular trends in absolute intakes, in both sexes, fibre intake decreased and added sugar

Table 1. Characteristics* at age at take-off (ATO) for 100 boys and 116 girls from the Dortmund Nutritional and Anthropometric Longitudinally Designed Study by calendar year strata

(Mean values and standard deviations, medians (first quartile (Q1) and third quartile (Q3)) or frequencies and percentages)

\begin{tabular}{|c|c|c|c|c|c|}
\hline \multirow[b]{3}{*}{ Variables } & \multicolumn{4}{|c|}{ Calendar year strata at ATO } & \multirow[b]{3}{*}{$P$ for trend } \\
\hline & \multicolumn{2}{|c|}{$1988-97(n$ 108) } & \multicolumn{2}{|c|}{$1998-03(n$ 108) } & \\
\hline & Mean/median & SD/Q1, Q3 & Mean/median & SD/Q1, Q3 & \\
\hline Boys & \multicolumn{2}{|c|}{39} & \multicolumn{2}{|c|}{61} & \\
\hline ATO (years) & $10 \cdot 2$ & $1 \cdot 1$ & $10 \cdot 3$ & 0.8 & 0.6 \\
\hline Age at PHV (years) & 13.5 & 1.0 & $13 \cdot 3$ & 0.9 & 0.3 \\
\hline Body fat $†(\%)$ & $13 \cdot 7$ & $11 \cdot 2,17 \cdot 6$ & $13 \cdot 9$ & $11 \cdot 8,21 \cdot 7$ & 0.3 \\
\hline BMI SDS & -0.08 & 0.88 & 0.15 & 0.97 & 0.2 \\
\hline Overweight $\ddagger$ & 4 & $10 \cdot 3$ & 11 & $18 \cdot 0$ & 0.3 \\
\hline Maternal overweight§ & 17 & $43 \cdot 6$ & 17 & $27 \cdot 9$ & $0 \cdot 1$ \\
\hline High maternal educational level\| & 19 & $48 \cdot 7$ & 36 & $59 \cdot 0$ & 0.3 \\
\hline Maternal age at ATO (years) & $39 \cdot 8$ & 3.4 & $41 \cdot 0$ & $4 \cdot 3$ & $0 \cdot 1$ \\
\hline Maximal four-person household & 35 & 89.7 & 55 & $90 \cdot 2$ & 0.9 \\
\hline Smoking in the household & 17 & $43 \cdot 6$ & 17 & $27 \cdot 9$ & $0 \cdot 1$ \\
\hline Girls & \multicolumn{2}{|c|}{69} & \multicolumn{2}{|c|}{47} & \\
\hline ATO (years) & $8 \cdot 6$ & 0.9 & $8 \cdot 8$ & 0.9 & 0.3 \\
\hline Age at PHV (years) & $11 \cdot 5$ & 0.9 & $11 \cdot 8$ & 0.9 & $0 \cdot 1$ \\
\hline Body fat $+(\%)$ & $18 \cdot 0$ & $15 \cdot 1,23 \cdot 3$ & $16 \cdot 4$ & $14 \cdot 3,22 \cdot 0$ & 0.2 \\
\hline BMI SDS & 0.16 & 0.94 & -0.07 & 0.96 & 0.2 \\
\hline Overweightł & 15 & $21 \cdot 7$ & 6 & $12 \cdot 8$ & 0.2 \\
\hline Maternal overweight§ & 24 & 34.8 & 16 & $34 \cdot 0$ & 0.9 \\
\hline High maternal educational level\| & 30 & 43.5 & 25 & $53 \cdot 2$ & 0.3 \\
\hline Maternal age at ATO (years) & 38.0 & $4 \cdot 8$ & 39.8 & $3 \cdot 0$ & 0.03 \\
\hline Maximal four-person household & 55 & $79 \cdot 7$ & 43 & 91.5 & 0.09 \\
\hline Smoking in the household & 28 & $40 \cdot 6$ & 15 & 31.9 & 0.3 \\
\hline
\end{tabular}

PHV, peak height velocity; SDS, standard deviation scores.

${ }^{*} P$ for trend was tested using ANOVA for normally distributed continuous variables, Kruskal-Wallis test for not normally distributed continuous variables and $\chi^{2}$ test for categorical variables.

† Calculated according to Slaughter et al. ${ }^{(49)}$.

$\ddagger$ Derived from the age- and sex-specific cut-off points proposed by the International Obesity Task Force, which are linked to the adult cut-off point of BMI of $25 \mathrm{~kg} / \mathrm{m}^{2(50)}$.

$\S B M I \geq 25 \mathrm{~kg} / \mathrm{m}^{2}$.

\|I School education for at least 12 years. 
Table 2. Dietary characteristics* of boys ( $n 100$, in total 486 dietary records) from the Dortmund Nutritional and Anthropometric Longitudinally Designed Study sample during the study period (Mean values and standard deviations)

\begin{tabular}{|c|c|c|c|c|c|c|c|c|c|c|c|c|c|c|}
\hline & \multicolumn{7}{|c|}{ Pubertal age $†$} & \multicolumn{7}{|c|}{ Time } \\
\hline & \multicolumn{2}{|c|}{ ATO } & \multicolumn{2}{|c|}{$\begin{array}{c}2 \text { Years after } \\
\text { ATO }\end{array}$} & \multicolumn{2}{|c|}{$\begin{array}{c}4 \text { Years after } \\
\text { ATO }\end{array}$} & \multirow[b]{2}{*}{$P$ for trend $\neq$} & \multicolumn{2}{|c|}{$1988-97$} & \multicolumn{2}{|c|}{ 1998-02 } & \multicolumn{2}{|c|}{$2003-7$} & \multirow[b]{2}{*}{$P$ for trenc } \\
\hline & Mean & SD & Mean & SD & Mean & SD & & Mean & SD & Mean & SD & Mean & SD & \\
\hline Number of records $(n)$ & \multicolumn{2}{|c|}{100} & \multicolumn{2}{|c|}{98} & \multicolumn{2}{|c|}{96} & & \multicolumn{2}{|c|}{118} & \multicolumn{2}{|c|}{244} & \multicolumn{2}{|c|}{124} & \\
\hline Absolute carbohydrate intake $(\mathrm{g} / \mathrm{d})$ & & & & & & & & & & & & & & \\
\hline $\mathrm{GL}$ & $132 \cdot 5$ & 29.9 & 144.3 & 37.4 & 173.0 & 41.5 & $<0.0001$ & $140 \cdot 1$ & 31.8 & $152 \cdot 0$ & 39.6 & $158 \cdot 1$ & 39.4 & 0.0008 \\
\hline Whole grain & 31.5 & $47 \cdot 4$ & $27 \cdot 1$ & 34.1 & $29 \cdot 6$ & $40 \cdot 9$ & 0.9 & 35.6 & $48 \cdot 3$ & $30 \cdot 6$ & 38.5 & $21 \cdot 0$ & $32 \cdot 1$ & 0.01 \\
\hline Fibre & $19 \cdot 8$ & 5.8 & $19 \cdot 9$ & 6.5 & $23 \cdot 9$ & $7 \cdot 8$ & $<0.0001$ & 21.9 & $6 \cdot 1$ & $20 \cdot 6$ & 6.5 & $20 \cdot 4$ & $7 \cdot 7$ & 0.2 \\
\hline Total added sugar & $62 \cdot 7$ & $32 \cdot 2$ & $75 \cdot 2$ & $40 \cdot 0$ & $86 \cdot 2$ & $44 \cdot 2$ & 0.0002 & $65 \cdot 2$ & 33.5 & $76 \cdot 0$ & 41.0 & $84 \cdot 1$ & $36 \cdot 7$ & 0.0006 \\
\hline From beverages & $18 \cdot 6$ & 20.9 & $20 \cdot 1$ & $26 \cdot 2$ & $30 \cdot 0$ & $32 \cdot 8$ & 0.04 & $17 \cdot 8$ & $20 \cdot 8$ & $26 \cdot 0$ & 28.5 & 23.5 & $24 \cdot 7$ & 0.02 \\
\hline From sweets & $26 \cdot 7$ & 17.5 & 30.5 & 23.4 & $28 \cdot 3$ & $19 \cdot 6$ & 0.1 & $30 \cdot 0$ & 17.9 & $28 \cdot 8$ & $22 \cdot 0$ & 31.5 & 22.5 & 0.5 \\
\hline From other sources§ & $17 \cdot 1$ & $10 \cdot 7$ & $20 \cdot 2$ & 14.0 & $28 \cdot 0$ & 21.4 & $<0.0001$ & $16 \cdot 8$ & 13.3 & $20 \cdot 7$ & 14.4 & $29 \cdot 0$ & $18 \cdot 6$ & $<0.0001$ \\
\hline Carbohydrate & $238 \cdot 1$ & 51.4 & $255 \cdot 7$ & $63 \cdot 0$ & 308.5 & $72 \cdot 6$ & $<0.0001$ & $250 \cdot 4$ & $55 \cdot 2$ & 269.6 & $67 \cdot 3$ & 279.9 & $66 \cdot 7$ & 0.002 \\
\hline \multicolumn{15}{|l|}{ Relative carbohydrate intake } \\
\hline GI\| & $55 \cdot 7$ & 3.8 & $56 \cdot 4$ & 3.5 & $56 \cdot 2$ & 4.0 & $0 \cdot 1$ & 55.9 & 3.8 & $56 \cdot 4$ & 3.7 & $56 \cdot 5$ & 3.9 & 0.5 \\
\hline GL (g/MJ) & 70.5 & 9.6 & $72 \cdot 1$ & 9.7 & $72 \cdot 9$ & $9 \cdot 8$ & 0.01 & $70 \cdot 3$ & 8.8 & $73 \cdot 3$ & $10 \cdot 3$ & 74.5 & $10 \cdot 5$ & 0.003 \\
\hline Whole grain (g/MJ) & 3.9 & $5 \cdot 7$ & $3 \cdot 2$ & $3 \cdot 8$ & $2 \cdot 9$ & 3.9 & 0.6 & $4 \cdot 3$ & $5 \cdot 7$ & 3.5 & $4 \cdot 2$ & $2 \cdot 2$ & $3 \cdot 1$ & 0.001 \\
\hline Fibre (g/MJ) & $2 \cdot 5$ & 0.6 & 2.4 & 0.6 & 2.4 & 0.6 & 0.4 & 2.6 & 0.6 & 2.4 & 0.6 & $2 \cdot 3$ & 0.6 & $<0.0001$ \\
\hline Total added sugar (\% E) q & $13 \cdot 2$ & $5 \cdot 8$ & $14 \cdot 8$ & $6 \cdot 8$ & 14.4 & $6 \cdot 8$ & 0.2 & $12 \cdot 9$ & $5 \cdot 8$ & 14.5 & $6 \cdot 8$ & $15 \cdot 8$ & $6 \cdot 3$ & 0.002 \\
\hline From beverages (\% E) & 3.8 & 3.9 & $4 \cdot 8$ & 4.9 & $5 \cdot 1$ & 5.4 & 0.4 & 3.6 & 3.9 & 4.9 & $5 \cdot 1$ & 4.5 & 4.6 & 0.049 \\
\hline From sweets (\% E) & 5.6 & 3.5 & $6 \cdot 0$ & 4.3 & $4 \cdot 7$ & 3.0 & 0.04 & $6 \cdot 0$ & 3.4 & 5.5 & 3.9 & 5.9 & 4.0 & 0.5 \\
\hline From other sources (\% E)§q & 3.6 & $2 \cdot 2$ & $4 \cdot 0$ & $2 \cdot 6$ & 4.6 & 3.5 & 0.04 & 3.3 & $2 \cdot 3$ & $4 \cdot 0$ & $2 \cdot 7$ & $5 \cdot 4$ & $3 \cdot 2$ & $<0.0001$ \\
\hline Carbohydrate (\% E)ף & $50 \cdot 6$ & 5.9 & $51 \cdot 2$ & $6 \cdot 3$ & 51.9 & $6 \cdot 0$ & 0.052 & $50 \cdot 2$ & $5 \cdot 2$ & $52 \cdot 1$ & $6 \cdot 5$ & $52 \cdot 7$ & $6 \cdot 1$ & 0.004 \\
\hline Energy $(\mathrm{MJ} / \mathrm{d})$ & 7.9 & 1.5 & $8 \cdot 3$ & 1.6 & 9.9 & 1.9 & $<0.0001$ & 8.3 & 1.4 & 8.7 & 1.9 & 8.9 & 1.8 & 0.04 \\
\hline
\end{tabular}

ATO, age at take-off; GL, glycaemic load; Gl, glycaemic index; \% E, \% energy.

*All nutritional data represent crude values.

† Dietary records at 1 year after ATO and 3 years after ATO were not considered in the table, but all dietary records $(n 486)$ were used for $P$ for trend.

$\ddagger P$ for trend was tested using PROC GLM.

$\S$ Sugar from other sources: the difference between the total added sugar and added sugar from beverages and sweets.

$\|$ GI was calculated using the glucose $=100$ scale.
१ Intakes of added sugar or carbohydrate $(\mathrm{g} / \mathrm{MJ})(\% \mathrm{E})=1.67$. 
Table 3. Dietary characteristics* of girls ( $n$ 116, in total 567 dietary records) from the Dortmund Nutritional and Anthropometric Longitudinally Designed Study sample during the study period (Mean values and standard deviations)

\begin{tabular}{|c|c|c|c|c|c|c|c|c|c|c|c|c|c|c|}
\hline & \multicolumn{7}{|c|}{ Pubertal age $\dagger$} & \multicolumn{7}{|c|}{ Time } \\
\hline & \multicolumn{2}{|c|}{ ATO } & \multicolumn{2}{|c|}{$\begin{array}{c}2 \text { Years after } \\
\text { ATO }\end{array}$} & \multicolumn{2}{|c|}{$\begin{array}{c}4 \text { Years after } \\
\text { ATO }\end{array}$} & \multirow[b]{2}{*}{$P$ for trend $\ddagger$} & \multicolumn{2}{|c|}{$1988-97$} & \multicolumn{2}{|c|}{ 1998-02 } & \multicolumn{2}{|c|}{$2003-7$} & \multirow[b]{2}{*}{$P$ for trend } \\
\hline & Mean & SD & Mean & SD & Mean & SD & & Mean & SD & Mean & SD & Mean & SD & \\
\hline Number of records $(n)$ & \multicolumn{2}{|c|}{116} & \multirow{2}{*}{\multicolumn{2}{|c|}{114}} & \multirow{2}{*}{\multicolumn{2}{|c|}{114}} & & \multirow{2}{*}{\multicolumn{2}{|c|}{241}} & \multirow{2}{*}{\multicolumn{2}{|c|}{231}} & \multirow{2}{*}{\multicolumn{2}{|c|}{95}} & \\
\hline Absolute carbohydrate intake $(\mathrm{g} / \mathrm{d})$ & & & & & & & & & & & & & & \\
\hline GL & $109 \cdot 2$ & 24.7 & $119 \cdot 0$ & $27 \cdot 2$ & 134.4 & 35.5 & $<0.0001$ & $116 \cdot 4$ & $27 \cdot 8$ & $123 \cdot 8$ & $31 \cdot 2$ & $124 \cdot 2$ & 32.5 & 0.01 \\
\hline Whole grain & $28 \cdot 2$ & 37.7 & $25 \cdot 8$ & $32 \cdot 4$ & 46.5 & $160 \cdot 7$ & 0.3 & 39.5 & 114.6 & 27.5 & $45 \cdot 3$ & $22 \cdot 8$ & $52 \cdot 9$ & $0 \cdot 1$ \\
\hline Fibre & $16 \cdot 9$ & 4.3 & $17 \cdot 1$ & $4 \cdot 8$ & $19 \cdot 3$ & 5.6 & 0.0005 & 18.4 & $5 \cdot 2$ & $17 \cdot 2$ & $5 \cdot 2$ & 17.9 & 4.4 & 0.02 \\
\hline Total added sugar & 53.7 & 24.8 & $62 \cdot 1$ & 29.4 & $68 \cdot 1$ & $35 \cdot 6$ & 0.005 & $58 \cdot 7$ & 29.2 & $64 \cdot 1$ & $32 \cdot 2$ & $61 \cdot 8$ & 31.6 & 0.2 \\
\hline From beverages & 14.0 & $15 \cdot 5$ & $18 \cdot 2$ & $21 \cdot 8$ & $21 \cdot 3$ & $25 \cdot 9$ & 0.06 & $16 \cdot 8$ & $19 \cdot 1$ & $19 \cdot 1$ & $20 \cdot 6$ & $17 \cdot 0$ & $23 \cdot 8$ & 0.4 \\
\hline From sweets & $25 \cdot 0$ & 14.1 & 27.5 & 17.4 & $27 \cdot 3$ & $19 \cdot 3$ & 0.7 & $26 \cdot 8$ & $17 \cdot 3$ & $28 \cdot 3$ & $18 \cdot 3$ & $22 \cdot 2$ & $14 \cdot 8$ & 0.02 \\
\hline From other sources§ & $14 \cdot 2$ & 9.5 & $16 \cdot 0$ & $12 \cdot 3$ & $19 \cdot 1$ & $13 \cdot 0$ & 0.001 & $14 \cdot 8$ & $10 \cdot 0$ & $16 \cdot 2$ & $12 \cdot 2$ & 21.9 & $12 \cdot 5$ & $<0.0001$ \\
\hline Carbohydrate & 195.8 & 40.7 & 211.3 & $44 \cdot 1$ & 237.0 & $59 \cdot 2$ & $<0.0001$ & $209 \cdot 6$ & $47 \cdot 2$ & $218 \cdot 2$ & $51 \cdot 0$ & 218.8 & $53 \cdot 6$ & 0.1 \\
\hline \multicolumn{15}{|l|}{ Relative carbohydrate intake } \\
\hline GI\| & $55 \cdot 7$ & 3.1 & $56 \cdot 2$ & 3.4 & $56 \cdot 6$ & 3.4 & 0.3 & 55.5 & $3 \cdot 1$ & $56 \cdot 6$ & 3.4 & $56 \cdot 7$ & 3.5 & 0.0003 \\
\hline $\mathrm{GL}(\mathrm{g} / \mathrm{MJ})$ & 71.6 & 8.8 & $72 \cdot 4$ & 9.9 & $74 \cdot 2$ & $11 \cdot 1$ & 0.3 & $70 \cdot 1$ & $9 \cdot 2$ & 74.6 & $10 \cdot 4$ & 73.3 & $10 \cdot 2$ & $<0.0001$ \\
\hline Whole grain (g/MJ) & 4.7 & $7 \cdot 0$ & 3.8 & 4.7 & $7 \cdot 1$ & 28.7 & 0.4 & $6 \cdot 0$ & $20 \cdot 1$ & $4 \cdot 1$ & 6.7 & 3.5 & 8.6 & 0.2 \\
\hline Fibre (g/MJ) & $2 \cdot 7$ & 0.6 & 2.5 & 0.6 & 2.6 & 0.7 & 0.4 & $2 \cdot 7$ & 0.7 & 2.5 & 0.7 & $2 \cdot 6$ & 0.6 & 0.02 \\
\hline Total added sugar (\% E)ף & 13.9 & 5.5 & $14 \cdot 8$ & $6 \cdot 0$ & $14 \cdot 7$ & $6 \cdot 4$ & 0.8 & 13.9 & $5 \cdot 8$ & $15 \cdot 2$ & $6 \cdot 4$ & $14 \cdot 2$ & $5 \cdot 4$ & 0.07 \\
\hline From beverages (\% E)ף & 3.5 & 3.7 & $4 \cdot 3$ & 4.9 & 4.5 & $5 \cdot 2$ & 0.5 & 3.8 & $4 \cdot 1$ & 4.5 & 4.7 & 3.7 & 4.7 & 0.2 \\
\hline From sweets (\% E) & $6 \cdot 6$ & 3.3 & $6 \cdot 6$ & 4.1 & 5.9 & 3.7 & 0.06 & 6.4 & 3.8 & $6 \cdot 8$ & $4 \cdot 0$ & $5 \cdot 2$ & 3.1 & 0.003 \\
\hline From other sources (\% E)§ף & 3.7 & $2 \cdot 4$ & 3.9 & $2 \cdot 7$ & $4 \cdot 2$ & $2 \cdot 7$ & 0.3 & 3.6 & $2 \cdot 4$ & 3.8 & $2 \cdot 6$ & $5 \cdot 1$ & $2 \cdot 6$ & $<0.0001$ \\
\hline Carbohydrate (\% E)ף & 51.4 & 5.4 & 51.5 & $6 \cdot 1$ & $52 \cdot 4$ & $6 \cdot 7$ & 0.6 & 50.5 & $5 \cdot 7$ & $52 \cdot 6$ & 5.9 & $51 \cdot 7$ & $6 \cdot 2$ & 0.0003 \\
\hline Energy $(\mathrm{MJ} / \mathrm{d})$ & 6.4 & 1.1 & $6 \cdot 9$ & 1.3 & $7 \cdot 6$ & 1.6 & $<0.0001$ & $7 \cdot 0$ & 1.4 & 6.9 & 1.4 & $7 \cdot 1$ & 1.4 & 0.7 \\
\hline
\end{tabular}

ATO, age at take-off; GL, glycaemic load; Gl, glycaemic index; \% E, \% energy.

†Dietary records at 1 year after ATO and 3 years after ATO were not considered in the table, but all dietary records $(n 567)$ were used for $P$ for trend. $¥ P$ for trend was tested using PROC GLM.

$\S$ Sugar from other source to added sugar and added sugar from beverages and sweets.

II Intakes of added sugar or carbohydrate (g/MJ) $(\% \mathrm{E})=1.67$. 
Table 4. Pubertal and secular trends* (per year) in carbohydrate quality (absolute intake) in 216 participants from the Dortmund Nutritional and Anthropometric Longitudinally Designed Study analysed by repeated-measures regression analysis

( $\beta$ coefficients with their standard errors)

\begin{tabular}{|c|c|c|c|c|c|c|c|c|c|c|c|c|}
\hline & \multicolumn{6}{|c|}{ Pubertal trend } & \multicolumn{6}{|c|}{ Secular trend } \\
\hline & \multicolumn{3}{|c|}{ Boys } & \multicolumn{3}{|c|}{ Girls } & \multicolumn{3}{|c|}{ Boys } & \multicolumn{3}{|c|}{ Girls } \\
\hline & $\beta$ & SE & $P$ & $\beta$ & SE & $P$ & $\beta$ & $P$ & SE & $\beta$ & SE & $P$ \\
\hline \multicolumn{13}{|l|}{$\mathrm{GL}(\mathrm{g} / \mathrm{d})$} \\
\hline Unadjusted model & 8.903 & $1 \cdot 164$ & $<0.0001$ & 5.805 & 0.856 & $<0.0001$ & 1.189 & 0.678 & 0.08 & 0.216 & 0.447 & 0.6 \\
\hline Adjusted model $†$ & 8.903 & $1 \cdot 163$ & $<0.0001$ & $5 \cdot 796$ & 0.856 & $<0.0001$ & $1 \cdot 188$ & 0.678 & 0.08 & 0.225 & 0.448 & 0.6 \\
\hline \multicolumn{13}{|l|}{ Fibre $(g / d)$} \\
\hline Unadjusted model & 0.984 & 0.198 & $<0.0001$ & 0.772 & 0.138 & $<0.0001$ & $-0 \cdot 190$ & 0.133 & 0.2 & -0.181 & 0.079 & 0.02 \\
\hline Adjusted model† & $0 \cdot 151$ & 0.195 & $<0.0001$ & 0.807 & 0.140 & $<0.0001$ & -0.358 & 0.129 & 0.006 & -0.216 & 0.081 & 0.008 \\
\hline \multicolumn{13}{|l|}{ Whole grain $(g / d)$} \\
\hline Unadjusted model & 0.300 & 1.255 & 0.8 & 0.204 & 1.493 & 0.9 & -0.922 & 0.609 & 0.1 & -0.760 & 0.555 & 0.2 \\
\hline Adjusted model $\dagger$ & 0.554 & 1.242 & 0.7 & 0.400 & 1.493 & 0.8 & $-1 \cdot 186$ & 0.580 & 0.04 & -0.962 & 0.554 & 0.08 \\
\hline \multicolumn{13}{|l|}{ Total added sugar (g/d) } \\
\hline Unadjusted model & $4 \cdot 274$ & 1.267 & 0.0008 & 3.397 & 0.809 & $<0.0001$ & 1.880 & 0.730 & 0.01 & 0.177 & 0.487 & 0.7 \\
\hline Adjusted model $\dagger$ & 4.239 & 1.263 & 0.001 & 3.469 & 0.801 & $<0.0001$ & 1.923 & 0.724 & 0.008 & 0.079 & 0.477 & 0.8 \\
\hline \multicolumn{13}{|l|}{ From beverage } \\
\hline Unadjusted model & 1.617 & 0.844 & 0.06 & 1.771 & 0.539 & 0.001 & 0.800 & 0.486 & 0.1 & 0.042 & 0.323 & 0.9 \\
\hline Adjusted model† & 1.582 & 0.830 & 0.06 & $1 \cdot 831$ & 0.535 & 0.001 & 0.849 & 0.462 & 0.07 & -0.019 & 0.316 & 0.9 \\
\hline \multicolumn{13}{|l|}{ From sweets } \\
\hline Unadjusted model & 0.957 & 0.633 & $0 \cdot 1$ & 0.503 & 0.488 & 0.3 & -0.159 & 0.369 & 0.7 & -0.285 & 0.231 & 0.2 \\
\hline Adjusted model† & 0.941 & 0.630 & 0.1 & 0.524 & 0.486 & 0.3 & -0.136 & 0.365 & 0.7 & -0.304 & 0.228 & 0.2 \\
\hline \multicolumn{13}{|l|}{ From other sources } \\
\hline Unadjusted model & 1.400 & 0.534 & 0.01 & 1.038 & 0.372 & 0.006 & 0.911 & 0.224 & $<0.0001$ & 0.349 & 0.146 & 0.02 \\
\hline Adjusted model $†$ & 1.385 & 0.533 & 0.01 & 1.004 & 0.371 & 0.007 & 0.927 & 0.222 & $<0.0001$ & 0.385 & 0.144 & 0.01 \\
\hline \multicolumn{13}{|l|}{ Carbohydrate $(\mathrm{g} / \mathrm{d})$} \\
\hline Unadjusted model & 14.58 & 1.950 & $<0.0001$ & $10 \cdot 23$ & 1.422 & $<0.0001$ & 1.989 & $1 \cdot 197$ & 0.09 & -0.048 & 0.735 & 0.9 \\
\hline Adjusted model† & 14.59 & 1.948 & $<0.0001$ & $10 \cdot 16$ & 1.422 & $<0.0001$ & 1.980 & $1 \cdot 193$ & $0 \cdot 1$ & -0.024 & 0.735 & 0.9 \\
\hline \multicolumn{13}{|l|}{ Energy $(\mathrm{MJ} / \mathrm{d})$} \\
\hline Unadjusted model & 0.444 & 0.052 & $<0.0001$ & 0.340 & 0.039 & $<0.0001$ & 0.019 & 0.033 & 0.6 & -0.030 & 0.020 & 0.1 \\
\hline Adjusted model $\dagger$ & 0.447 & 0.053 & $<0.0001$ & 0.337 & 0.039 & $<0.0001$ & 0.016 & 0.033 & 0.6 & -0.027 & 0.020 & 0.2 \\
\hline
\end{tabular}

GL, glycaemic load; ATO, age at take-off; SDS, standard deviation scores.

*Pubertal trend, directly from ATO and over the subsequent 4 years; secular trend, from year 1988 to year 2007.

†BMI SDS at ATO, maternal education and maternal overweight were included in the adjusted model, since they significantly affected the pubertal and secular trends of the carbohydrate quality in the basic models. Models of fibre intake and whole-grain intake were adjusted additionally for BMI SDS at ATO, maternal education and maternal overweight; models of total added sugar and its subgroups, dietary GL and carbohydrate intake were adjusted additionally for BMI SDS at ATO; energy model was adjusted additionally for BMI SDS at ATO and maternal overweight.

from other sources increased between 1988 and 2007 (Table 4, second set of columns). In boys, the absolute whole-grain intake decreased, and added sugar intake increased over time.

The only pubertal trends for relative $\mathrm{CHO}$ intakes were an increase in added sugar intake from beverages in girls and a decrease in added sugar intake from sweets in both sexes (Table 5, first set of columns). With respect to secular trends, the relative intakes of $\mathrm{CHO}$, GL and added sugar from other sources increased over time (Table 5, second set of columns). Downward secular trends in fibre and wholegrain intake densities and upward secular trends in total added sugar were only observed in boys.

\section{Discussion}

The present study suggests that in the population of healthy adolescents, the $\mathrm{CHO}$ quality did not change considerably over the course of puberty. However, $\mathrm{CHO}$ quality declined over the last 20 years, i.e. absolute fibre intake decreased and the contribution of added sugar from sources other than beverages and sweets to total energy intake increased. These adverse 20-year secular trends were most pronounced in boys, with an additional increase in total added sugar intake and decreases in fibre and whole-grain intakes.
During puberty, adolescents increase their self-awareness and are exposed to a changing lifestyle, all of which may affect their eating behaviours ${ }^{(34)}$, e.g. fast food and soft drink consumption increases ${ }^{(2)}$. In the present study, girls increased their energy intake from added sugar provided by beverages during puberty, corresponding to an increase of $1 \%$ in energy over 5 years. Excess consumption of soft drinks is considered a risk factor influencing body composition ${ }^{(35,36)}$. However, girls concomitantly decreased their energy intake from added sugar provided by sweets, hence compensating for their increase in added sugar from beverages. Therefore, increases in soft drink consumption during puberty may be less important than commonly assumed.

In the present study, relative $\mathrm{CHO}$ intakes increased over the 20-year observational period. The concomitant increase in GL was primarily driven by this increase in total $\mathrm{CHO}$ and to a lesser extent by an upward secular trend in GI. The present results for adolescents confirm and extend a previous analysis in 7- to 8-year-old DONALD participants, where the GI and GL increased moderately between 1990 and $2002^{(24)}$.

The present study indicates that $\mathrm{CHO}$ quality declined remarkably over the past 20 years. Between 1988 and 2007, absolute fibre intakes decreased considerably in both sexes, corresponding to a $10-15 \%$ decrease over a 10 -year 
Table 5. Pubertal and secular trends* (per year) in carbohydrate quality (relative intake) in 216 participants from the Dortmund Nutritional and Anthropometric Longitudinally Designed Study analysed by repeated-measures regression analysis

( $\beta$ coefficients with their standard errors)

\begin{tabular}{|c|c|c|c|c|c|c|c|c|c|c|c|c|}
\hline & \multicolumn{6}{|c|}{ Pubertal trend } & \multicolumn{6}{|c|}{ Secular trend } \\
\hline & \multicolumn{3}{|c|}{ Boys } & \multicolumn{3}{|c|}{ Girls } & \multicolumn{3}{|c|}{ Boys } & \multicolumn{3}{|c|}{ Girls } \\
\hline & $\beta$ & SE & $P$ & $\beta$ & SE & $P$ & $\beta$ & SE & $P$ & $\beta$ & SE & $P$ \\
\hline \multicolumn{13}{|l|}{ GI } \\
\hline Unadjusted model & $0 \cdot 123$ & $0 \cdot 114$ & 0.3 & $0 \cdot 103$ & 0.097 & 0.3 & 0.079 & 0.071 & 0.3 & 0.102 & 0.051 & 0.049 \\
\hline Adjusted model $\dagger$ & 0.066 & 0.119 & 0.6 & 0.076 & 0.096 & 0.4 & 0.115 & 0.073 & 0.1 & 0.097 & 0.052 & 0.06 \\
\hline \multicolumn{13}{|l|}{$\mathrm{GL}(\mathrm{g} / \mathrm{MJ})$} \\
\hline Unadjusted model & $0 \cdot 102$ & 0.077 & 0.2 & 0.011 & 0.073 & 0.9 & 0.115 & 0.040 & 0.004 & 0.102 & 0.037 & 0.006 \\
\hline Adjusted model $\dagger$ & $0 \cdot 101$ & 0.077 & 0.2 & 0.012 & 0.073 & 0.9 & 0.115 & 0.040 & 0.004 & $0 \cdot 100$ & 0.037 & 0.007 \\
\hline \multicolumn{13}{|l|}{ Fibre $(g / M J)$} \\
\hline Unadjusted model & 0.001 & 0.018 & 0.9 & -0.008 & 0.018 & 0.7 & -0.033 & 0.013 & 0.01 & -0.012 & 0.011 & 0.3 \\
\hline Adjusted model $\dagger$ & 0.008 & 0.018 & 0.6 & -0.007 & 0.018 & 0.7 & -0.040 & 0.012 & 0.001 & -0.012 & 0.011 & 0.2 \\
\hline \multicolumn{13}{|l|}{ Whole grain (g/MJ) } \\
\hline Unadjusted model & -0.111 & 0.144 & 0.4 & -0.219 & 0.253 & 0.4 & -0.122 & 0.064 & 0.06 & -0.106 & 0.078 & 0.2 \\
\hline Adjusted model $\dagger$ & -0.094 & 0.142 & 0.5 & -0.184 & 0.253 & 0.5 & -0.141 & 0.059 & 0.02 & -0.140 & 0.077 & 0.07 \\
\hline \multicolumn{13}{|c|}{ Total added sugar (\% E) $\ddagger$} \\
\hline Unadjusted model & 0.154 & 0.220 & 0.5 & 0.097 & 0.163 & 0.6 & 0.308 & 0.128 & 0.02 & 0.063 & $0 \cdot 100$ & 0.5 \\
\hline Adjusted model $\dagger$ & 0.145 & 0.219 & 0.5 & $0 \cdot 122$ & $0 \cdot 161$ & 0.4 & 0.320 & 0.124 & 0.01 & 0.039 & 0.097 & 0.7 \\
\hline \multicolumn{13}{|l|}{ From beverages $(\% \mathrm{E}) \ddagger$} \\
\hline Unadjusted model & 0.164 & $0 \cdot 151$ & 0.3 & 0.235 & 0.121 & 0.05 & 0.125 & 0.089 & 0.2 & 0.004 & 0.074 & 0.9 \\
\hline Adjusted model† & 0.133 & 0.147 & 0.4 & 0.247 & 0.119 & 0.04 & 0.157 & 0.083 & 0.06 & 0.009 & 0.072 & 0.9 \\
\hline \multicolumn{13}{|l|}{ From sweets (\% E)‡ } \\
\hline Unadjusted model & -0.219 & $0 \cdot 114$ & 0.06 & -0.200 & 0.098 & 0.04 & -0.018 & 0.067 & 0.8 & -0.043 & 0.042 & 0.3 \\
\hline Adjusted model† & -0.222 & 0.113 & 0.049 & -0.200 & 0.098 & 0.04 & -0.013 & 0.066 & 0.8 & -0.040 & 0.041 & 0.3 \\
\hline \multicolumn{13}{|c|}{ From other sources $(\% \mathrm{E}) \ddagger$} \\
\hline Unadjusted model & 0.051 & 0.096 & 0.6 & 0.052 & 0.084 & 0.5 & 0.191 & 0.044 & $<0.0001$ & 0.106 & 0.034 & 0.002 \\
\hline Adjusted model $\dagger$ & 0.048 & 0.096 & 0.6 & 0.046 & 0.084 & 0.6 & 0.194 & 0.044 & $<0.0001$ & 0.113 & 0.034 & 0.001 \\
\hline \multicolumn{13}{|l|}{ Carbohydrate $(\% \mathrm{E}) \ddagger$} \\
\hline Unadjusted model & 0.241 & 0.206 & 0.2 & -0.059 & 0.184 & 0.8 & 0.230 & 0.110 & 0.03 & 0.199 & 0.093 & 0.03 \\
\hline Adjusted model $†$ & 0.241 & 0.206 & 0.2 & -0.064 & 0.184 & 0.7 & 0.230 & 0.111 & 0.04 & 0.204 & 0.099 & 0.03 \\
\hline
\end{tabular}

GI, glycaemic index; GL, glycaemic load; \% E, \% energy; ATO, age at take-off; SDS, standard deviation scores.

* Pubertal trend, directly from ATO and over the subsequent 4 years; secular trend, from year 1988 to year 2007.

†BMI SDS at ATO, maternal education and maternal overweight were included in the adjusted model, since they significantly affected the pubertal and secular trends of the carbohydrate quality in the basic models. Models of dietary GI and intakes of fibre and whole grain were adjusted additionally for BMI SDS at ATO, maternal education and maternal overweight; models of total added sugar and its subgroups, dietary GL and carbohydrate intake were adjusted additionally for BMI SDS at ATO.

$\ddagger$ Intakes of added sugar or carbohydrate $(\mathrm{g} / \mathrm{MJ})(\% \mathrm{E})=1.67$.

time period. This decline was more pronounced in boys who concomitantly decreased their fibre and whole-grain densities by -0.4 and $-1.4 \mathrm{~g} / \mathrm{MJ}$, respectively, in 10 years. Some ${ }^{(6,37)}$, but not all ${ }^{(38,39)}$, previous trend analyses of $\mathrm{CHO}$ intakes in adolescents support a reduction in fibre intake: daily fibre intakes decreased by $1.15 \mathrm{~g} / \mathrm{d}$ in 12 - to 18 -year-old US boys between $1977-8$ and $1987-8^{(37)}$, and fibre intakes decreased by $0.18 \mathrm{~g} / \mathrm{d}$ in German girls aged 9-13 years between 1990 and $2004^{(6)}$. In view of the numerous recognised health benefits associated with higher intakes of fibre and whole grains ${ }^{(40)}$, these marked downward secular trends in adolescents are a cause for concern, since they may result in an increased risk for diabetes mellitus, CVD and cancer in adulthood.

In the present study, boys presented with an upward secular trend in added sugar intake, amounting to $3 \%$ more energy intake in 10 years. High consumption of added sugars is thought to be associated with a decrease in micronutrient density in children and teenagers ${ }^{(7)}$. Furthermore, we observed secular upward trends in the consumption of added sugar from sources other than beverages and sweets for both sexes. Further analysis revealed that this category consisted mainly of breakfast cereals and pastries (data not shown).

Interestingly, the adverse secular trends were less pronounced in girls. Females have been suggested to commonly give greater relevance to healthy eating and to make healthier food choices, e.g. they consume more fibre and less fat ${ }^{(41)}$. In addition, attention to weight control is more prominent in adolescent girls, and they are more likely to diet or restrain their eating behaviour ${ }^{(42)}$. Furthermore, females are more likely to under-report their dietary intake ${ }^{(43)}$. However, in the present study, neither exclusion of dietary records with potentially under-reported energy levels $(3 \%$ of the observations in our girls were under-reported according to age-specific cut-off points ${ }^{(15)}$ ) nor adjustment for the ratio of reported energy intake to estimated BMR appreciably altered the findings. Nevertheless, selective under-reporting of socially undesirable foods such as sweets and soft drinks or selective over-reporting of healthy foods such as fruits and vegetables might have attenuated the secular and pubertal trends in $\mathrm{CHO}$ intake, particularly among girls.

The fact that GI values had to be calculated for approximately $35 \%$ of the CHO-containing foods is a limitation of the present study. While this procedure has been controversially discussed ${ }^{(44)}$, several reports suggest that the GI of a whole diet or mixed meal can be accurately estimated from the GI values of its ingredients ${ }^{(45,46)}$. Furthermore, whenever no GI or a close match is available for a food, its calculation from the ingredients is the only feasible approach for epidemiological studies. In addition, the high correlation between 
GL and CHO is in line with the previously expressed concern that GL estimates are largely 'surrogates' for CHO intake ${ }^{(47)}$. Theoretically, a validation of GI and GL values could provide further insights in this regard; however, validation of estimates obtained from weighed dietary records would require repeated measurements of postprandial blood glucose levels, an approach which is not feasible particularly in an observational study with children and adolescents.

Given the relatively small study sample, the possibility of chance findings cannot be excluded. The elaborate design of the DONALD Study resulting in a study sample with a relatively high socio-economic status represents a further limitation of the study. While non-representativeness is less relevant for the interpretation of the pubertal trends since each individual acts as his or her own control, it does limit the generalisability of our secular trends. However, the $\mathrm{CHO}$ intake of our participants $(49-52 \%$ energy) was similar to the values observed in other studies in adolescents ${ }^{(5,38)}$, and the added sugar intake (13-15\% energy) was in line with the values found in a representative German study ${ }^{(48)}$. The fact that our adolescents consumed more fibre than adolescents in other studies ${ }^{(5,38)}$ suggests that our secular trends may underestimate the 'true' decline in $\mathrm{CHO}$ quality over the past 20 years in the general population of European adolescents.

The present study has several strengths, including its comprehensive analysis of various aspects of $\mathrm{CHO}$ quality and the use of an innovative statistical approach ${ }^{(32)}$ to simultaneously address independent trends during puberty and over the time. The prospectively collected, repeated and detailed measurements of anthropometric and dietary data for each participant allowed us to align the present analysis to a physiological marker of early puberty so as to reliably estimate individual pubertal trends in $\mathrm{CHO}$ quality. A further advantage lies in the constantly updated nutritional database, Lebensmitteltabelle (LEBTAB), which is a prerequisite for a precise evaluation of 20-year secular trends.

In conclusion, the quality of dietary $\mathrm{CHO}$ consumed by healthy German adolescents did not change considerably over the course of puberty. However, public health initiatives should be tailored at improving the overall quality of $\mathrm{CHO}$ nutrition, which appears to have declined notably over the past 20 years, especially among boys.

\section{Acknowledgements}

The authors thank the staff of the Research Institute of Child Nutrition for carrying out the anthropometric measurements and for carrying out the medical examinations. The authors' contributions are as follows: G. C., A. E. B. and L. L. conceived the project, performed the data analyses and drafted the manuscript. N. K.-D., U. A. and T. R. provided critical input on the data analyses and on earlier versions of the manuscript. K. B. provided technical support and statistical expertise. A. E. B. supervised the study. All authors contributed to the interpretation of the data and revision of the manuscript. The DONALD Study is funded by the Ministry of Science and Research of North Rhine Westphalia, Germany. The present analysis was funded by the International Foundation for the Promotion of Nutrition Research and Nutrition Education. None of the authors has any personal or financial conflicts of interest.

\section{References}

1. Lytle LA, Seifert S, Greenstein J, et al. (2000) How do children's eating patterns and food choices change over time? Results from a cohort study. Am J Health Promot 14, 222-228.

2. St-Onge MP, Keller KL \& Heymsfield SB (2003) Changes in childhood food consumption patterns: a cause for concern in light of increasing body weights. Am J Clin Nutr 78, 1068-1073.

3. French SA, Story M, Neumark-Sztainer D, et al. (2001) Fast food restaurant use among adolescents: associations with nutrient intake, food choices and behavioral and psychosocial variables. Int J Obes Relat Metab Disord 25, 1823-1833.

4. Alexy U, Sichert-Hellert W \& Kersting M (2002) Fifteen-year time trends in energy and macronutrient intake in German children and adolescents: results of the DONALD study. $\mathrm{Br} \mathrm{J}$ Nutr 87, 595-604.

5. Nicklas TA, Elkasabany A, Srinivasan SR, et al. (2001) Trends in nutrient intake of 10-year-old children over two decades (1973-1994): the Bogalusa Heart Study. Am J Epidemiol 153, 969-977.

6. Alexy U, Kersting M \& Sichert-Hellert W (2006) Evaluation of dietary fibre intake from infancy to adolescence against various references - results of the DONALD study. Eur J Clin Nutr 60, 909-914.

7. Joyce $\mathrm{T} \&$ Gibney MJ (2008) The impact of added sugar consumption on overall dietary quality in Irish children and teenagers. J Hum Nutr Diet 21, 438-450.

8. Dietz WH (1994) Critical periods in childhood for the development of obesity. Am J Clin Nutr 59, 955-959.

9. Kroke A, Manz F, Kersting M, et al. (2004) The DONALD study: history, current status and future perspectives. Eur $J$ Nutr 43, 45-54.

10. Euling SY, Herman-Giddens ME, Lee PA, et al. (2008) Examination of US puberty-timing data from 1940 to 1994 for secular trends: panel findings. Pediatrics 121, Suppl. 3, S172-S191.

11. Mirwald RL, Baxter-Jones AD, Bailey DA, et al. (2002) An assessment of maturity from anthropometric measurements. Med Sci Sports Exerc 34, 689-694.

12. Silventoinen K, Haukka J, Dunkel L, et al. (2008) Genetics of pubertal timing and its associations with relative weight in childhood and adult height: the Swedish Young Male Twins Study. Pediatrics 121, e885-e891.

13. Sandhu J, Ben-Shlomo Y, Cole TJ, et al. (2006) The impact of childhood body mass index on timing of puberty, adult stature and obesity: a follow-up study based on adolescent anthropometry recorded at Christ's Hospital (1936-1964). Int J Obes (Lond) 30, 14-22.

14. He Q \& Karlberg J (2001) Bmi in childhood and its association with height gain, timing of puberty, and final height. Pediatr Res 49, 244-251.

15. Sichert-Hellert W, Kersting M \& Schoch G (1998) Underreporting of energy intake in 1 to 18 year old German children and adolescents. $Z$ Ernahrungswiss 37, 242-251.

16. Lohman TG, Roche AF \& Martorell R (1988) Anthropometric Standardization Reference Manual. Champaign, IL: Human Kinetics Publishers.

17. Preece MA \& Baines MJ (1978) A new family of mathematical models describing the human growth curve. Ann Hum Biol 5, $1-24$.

18. Buyken AE, Karaolis-Danckert N \& Remer T (2009) Association of prepubertal body composition in healthy girls and boys with the timing of early and late pubertal markers. Am $J$ Clin Nutr 89, 221-230.

19. Berkey CS, Dockery DW, Wang X, et al. (1993) Longitudinal height velocity standards for U.S. adolescents. Stat Med 12, 403-414. 
20. Demerath EW, Li J, Sun SS, et al. (2004) Fifty-year trends in serial body mass index during adolescence in girls: the Fels Longitudinal Study. Am J Clin Nutr 80, 441-446.

21. Aksglaede L, Olsen LW, Sorensen TI, et al. (2008) Forty years trends in timing of pubertal growth spurt in 157,000 Danish school children. PLoS One 3, e2728.

22. Human Nutrition Unit, School of Molecular and Microbial Biosciences and University of Sydney (2007) www.glycemicindex. com (accessed 25 September 2007).

23. Sichert-Hellert W, Kersting M, Chahda C, et al. (2007) German food composition database for dietary evaluations in children and adolescents. J Food Compost Anal 20, 63-70.

24. Buyken AE, Dettmann W, Kersting M, et al. (2005) Glycaemic index and glycaemic load in the diet of healthy schoolchildren: trends from 1990 to 2002, contribution of different carbohydrate sources and relations to dietary quality. Br J Nutr 94, 796-803.

25. Cheng G, Karaolis-Danckert N, Libuda L, et al. (2009) Relation of dietary glycemic index, glycemic load, and fiber and wholegrain intakes during puberty to the concurrent development of percent body fat and body mass index. Am J Epidemiol 169, 667-677.

26. U.S. Department of Health and Human Services and U.S. Department of Agriculture (2005) www.healthierus.gov/dietaryguidelines (accessed 17 March 2010).

27. Buyken AE, Cheng G, Gunther AL, et al. (2008) Relation of dietary glycemic index, glycemic load, added sugar intake, or fiber intake to the development of body composition between ages 2 and $7 \mathrm{y}$. Am J Clin Nutr 88, 755-762.

28. Lytle LA \& Kubik MY (2003) Nutritional issues for adolescents. Best Pract Res Clin Endocrinol Metab 17, 177-189.

29. Ludwig DS, Peterson KE \& Gortmaker SL (2001) Relation between consumption of sugar-sweetened drinks and childhood obesity: a prospective, observational analysis. Lancet 357, 505-508.

30. Bingham SA, Cassidy A, Cole TJ, et al. (1995) Validation of weighed records and other methods of dietary assessment using the $24 \mathrm{~h}$ urine nitrogen technique and other biological markers. Br J Nutr 73, 531-550.

31. Bokhof B, Gunther AL, Berg-Beckhoff G, et al. (2010) Validation of protein intake assessed from weighed dietary records against protein estimated from $24 \mathrm{~h}$ urine samples in children, adolescents and young adults participating in the Dortmund Nutritional and Longitudinally Designed (DONALD) Study. Public Health Nutr 13, 826-834.

32. Jacobs DR Jr, Hannan PJ, Wallace D, et al. (1999) Interpreting age, period and cohort effects in plasma lipids and serum insulin using repeated measures regression analysis: the CARDIA Study. Stat Med 18, 655-679.

33. Hulshof KF, Brussaard JH, Kruizinga AG, et al. (2003) Socioeconomic status, dietary intake and $10 \mathrm{y}$ trends: the Dutch National Food Consumption Survey. Eur J Clin Nutr 57, $128-137$.
34. Rolland-Cachera MF, Bellisle F \& Deheeger M (2000) Nutritional status and food intake in adolescents living in Western Europe. Eur J Clin Nutr 54, Suppl. 1, S41-S46.

35. Malik VS, Willett WC \& Hu FB (2009) Sugar-sweetened beverages and BMI in children and adolescents: reanalyses of a meta-analysis. Am J Clin Nutr 89, 438-439, author reply 439-440.

36. Vartanian LR, Schwartz MB \& Brownell KD (2007) Effects of soft drink consumption on nutrition and health: a systematic review and meta-analysis. Am J Public Health 97, 667-675.

37. Saldanha LG (1995) Fiber in the diet of US children: results of national surveys. Pediatrics 96, 994-997.

38. Cavadini C, Siega-Riz AM \& Popkin BM (2000) US adolescent food intake trends from 1965 to 1996. Arch Dis Child 83, $18-24$.

39. Nicklas TA, Farris RP, Myers L, et al. (1995) Dietary fiber intake of children and young adults: the Bogalusa Heart Study. $J$ Am Diet Assoc 95, 209-214.

40. Slavin JL (2008) Position of the American Dietetic Association: health implications of dietary fiber. J Am Diet Assoc 108 $1716-1731$.

41. Wardle J, Haase AM, Steptoe A, et al. (2004) Gender differences in food choice: the contribution of health beliefs and dieting. Ann Behav Med 27, 107-116.

42. Neumark-Sztainer D, Story M, Resnick MD, et al. (1998) Lessons learned about adolescent nutrition from the Minnesota Adolescent Health Survey. J Am Diet Assoc 98, 1449-1456.

43. Novotny JA, Rumpler WV, Riddick H, et al. (2003) Personality characteristics as predictors of underreporting of energy intake on 24-hour dietary recall interviews. J Am Diet Assoc 103, $1146-1151$.

44. Hollenbeck CB \& Coulston AM (1991) The clinical utility of the glycemic index and its application to mixed meals. Can J Physiol Pharmacol 69, 100-107.

45. Wolever TM, Yang M, Zeng XY, et al. (2006) Food glycemic index, as given in glycemic index tables, is a significant determinant of glycemic responses elicited by composite breakfast meals. Am J Clin Nutr 83, 1306-1312.

46. Chew I, Brand JC, Thorburn AW, et al. (1988) Application of glycemic index to mixed meals. Am J Clin Nutr 47, 53-56.

47. van Bakel MM, Slimani N, Feskens EJ, et al. (2009) Methodological challenges in the application of the glycemic index in epidemiological studies using data from the European Prospective Investigation into Cancer and Nutrition. J Nutr 139, 568-575.

48. Linseisen J, Gedrich K, Karg G, et al. (1998) Sucrose intake in Germany. Z Ernahrungswiss 37, 303-314.

49. Slaughter MH, Lohman TG, Boileau RA, et al. (1988) Skinfold equations for estimation of body fatness in children and youth. Hum Biol 60, 709-723.

50. Cole TJ, Bellizzi MC, Flegal KM, et al. (2000) Establishing a standard definition for child overweight and obesity worldwide: international survey. BMJ 320, 1240-1243. 